\title{
CHANGE OF VARIABLES AS BOREL RESUMMATION OF SEMICLASSICAL SERIES
}

\author{
Stefan Giller㔹 and Piotr Milczarski把 \\ Theoretical Physics Department II, University of Łódź, \\ Pomorska 149/153, 90-236 Łódź, Poland \\ e-mail: † sgiller@krysia.uni.lodz.pl \\ † jezykmil@krysia.uni.lodz.pl
}

\begin{abstract}
It is shown that a change of variable in 1-dim Schrödinger equation applied to the Borel summable fundamental solutions [8, 9] is equivalent to Borel resummation of the fundamental solutions multiplied by suitably chosen $\hbar$-dependent constant. This explains why change of variable can improve JWKB formulae [11]. It is shown also that a change of variable alone cannot provide us with the exact JWKB formulae.
\end{abstract}

PACS number(s): 03.65.-W , 03.65.Sq, 02.30.Lt, 02.30.Mv

Key Words: Schrödinger equation, fundamental solutions, Maslov theory, change of variable, semiclasical expansion, Borel summability.

\footnotetext{
${ }^{1}$ Supported by KBN 2PO3B 07610

${ }^{2}$ Supported by the Eódź University Grant No 580
} 


\section{Introduction}

A change of variable in the 1-dim Schrödinger equation (1-DSE) is one of the basic technic used to solve 1-dim problems (see [12] for example). In the context of semiclassical (JWKB) approximation the procedure is in fact a main ingredient of Fröman and Fröman (F-F) approach to 1-DSE [3, 4] with an aim of getting improved JWKB quantization formulae [4, 5, 6]. Sometimes, a suitable change of variable provides us with JWKB-like formulae solving the problem of energy spectra even exactly [4]. No doubts, however, that the latter possibility depends totally on a potential considered and changing variable plays in such cases only an auxiliary role [1].

A change of variables is also an essential ingredient of a more general approach to the semiclassical approximations formulated by Maslov and his collaborators [13]. In the context of the latter approach the change-of-variable procedure is an inherent part of the continuation procedure of semiclassical series defined originally in some domain of the configuration space to another domain of the space. The relevant variable transformations used in the Maslov method are the canonical ones (in a sens of classical mechanics). A relation of the Maslov method to the one applied in this paper is discussed in Appendix. It is argued there however that using fundamental solutions as we do in our paper is equivalent to the method of Maslov et al in the semiclassical regime of the considered 1-dim problems but has many advantages over the Maslov procedure in the remaining of our investigations. In particular the problem of Borel resummation central for our paper cannot be put and considered properly ignoring the existence of the fundamental solutions and their properties. After all the method of Maslov et al is purely asymptotic from the very begining and any problem of resummation of the semiclassical series used in the method has not been considered as yet.

An improvement of the standard JWKB formulae achieved by the changing variable procedure appears as corrections having typically forms of additional $\hbar$-dependent term in emerging effective potentials [1, 4, 5, 6]. Since in all these cases of variable changing the standard JWKB formulae can be easily restored simply by $\hbar$-expansions of the improved ones the latter seems to be a kind of some hidden resummation of a part (in the case of improvements only) or a full (when exact formulae emerge) standard semiclassical expansion corresponding to considered cases.

It is the aim of this paper to show that indeed this hidden resummation mentioned above really takes place and that a class of applied changes of variable in 1-DSE results as the Borel resummation of suitably chosen standard semiclassical solutions to SE multiplied by appropriately chosen $\hbar$-dependent constants which can always be attached to any of such semiclassical solution.

As it has been shown by Milczarski and Giller [7] (see also [8]) such specific Borel summable solutions to SE are provided for meromorphic potentials by the F-F construction [3] in the form of so called fundamental solutions (FS) [8, 9]. These are the only solutions with the Borel summability property among all the F-F-like solutions [7]. Despite their rareness the FS's when collected into a full set of them allow us to solve any 1-dim problem [8, 9] (see also a discussion below).

The paper is organized as follows.

In the next section the fundamental solutions and their use are recalled.

In Sec. 3 the standard semiclassical expansions and their properties are reconsidered.

In Sec.4 the Borel resummation aspects of a change-of-variable operation are discussed. 
In Sec. 5 the impossibility of achieving the exact JWKB formulae by a change-of-variable operation only is discussed.

We conclude with Sec.6.

\section{$2 \quad$ Fundamental solutions}

A standard way to introduce FS's is a construction of a Stokes graph (SG) [7, 8, 9] for a given (meromorphic) potential $V(x)$.

SG consists of Stokes lines (SL) emerging from roots (turning points) of the equation:

$$
V(x)+\hbar^{2} \delta(x)=E
$$

with $E$ as energy as well as from simple poles of the considered potential $V(x)$.

The presence and role of the $\delta$-term in (2.1) is explained below. It contributes to (2.1) only when $V(x)$ contains simple and second order poles. The $\delta$-term is constructed totally from these poles.

The points of SL's satisfy one of the following equations:

$$
\Re \int_{x_{i}}^{x} \sqrt{V(y)+\hbar^{2} \delta(y)-E} d y=0
$$

with $x_{i}$ being a root of (2.1) or a simple pole of $V(x)$.

SL's which are not closed end at these points of the $x$-plane (i.e. have the latter as the boundaries) for which an action integral in (2.2) becomes infinite. Of course such points are singular for the potential $V(x)$ and can be finite poles, higher than the simple ones, or poles of $V(x)$ lying at the infinity.

Each such a singularity $x_{0}$ of $V(x)$ defines a domain called a sector. This is the connected domain of the x-plane bounded by the SL's and $x_{0}$ itself with the latter point being also a boundary for the SL's or being an isolated boundary point of the sector (as it is in the case of the second order pole).

In each sector the LHS in (2.2) is only positive or negative.

Consider now the Schrödinger equation:

$$
\Psi^{\prime \prime}(x)-\hbar^{-2} q(x) \Psi(x)=0
$$

where $q(x)=V(x)-E$ (we have put the mass $\mathrm{m}$ in (2.3) to be equal to $1 / 2$ ).

Following Fröman and Fröman one can define in each sector $k$ having $x_{0}$ at its boundary a solution of the form:

$$
\Psi_{k}(x)=\tilde{q}^{-\frac{1}{4}}(x) \cdot e^{\frac{\sigma}{\hbar} W(x)} \cdot \chi_{k}(x) \quad k=1,2, \ldots
$$

where:

$$
\begin{gathered}
\chi_{k}(x)=1+\sum_{n \geq 1}\left(-\frac{\sigma \hbar}{2}\right)^{n} \int_{x_{0}}^{x} d \xi_{1} \int_{x_{0}}^{\xi_{1}} d \xi_{2} \ldots \int_{x_{0}}^{\xi_{n-1}} d \xi_{n} \omega\left(\xi_{1}\right) \omega\left(\xi_{2}\right) \ldots \omega\left(\xi_{n}\right) \\
\times\left(1-e^{-\frac{2 \sigma}{\hbar}\left(W(x)-W\left(\xi_{1}\right)\right)}\right)\left(1-e^{-\frac{2 \sigma}{\hbar}\left(W\left(\xi_{1}\right)-W\left(\xi_{2}\right)\right)}\right) \cdots\left(1-e^{-\frac{2 \sigma}{\hbar}\left(W\left(\xi_{n-1}\right)-W\left(\xi_{n}\right)\right)}\right)
\end{gathered}
$$


with

$$
\omega(x)=\frac{\delta(x)}{\tilde{q}^{\frac{1}{2}}(x)}-\frac{1}{4} \frac{\tilde{q}^{\prime \prime}}{\tilde{q}^{\frac{3}{2}}(x)}+\frac{5}{16} \frac{\tilde{q}^{\prime 2}}{\tilde{q}^{\frac{5}{2}}(x)}
$$

and

$$
\begin{gathered}
W(x, E)=\int_{x_{i}}^{x} \sqrt{\tilde{q}(\xi, E)} d \xi \\
\tilde{q}(x, E)=V(x)+\hbar^{2} \delta(x)-E
\end{gathered}
$$

In (2.4) and (2.5) a sign $\sigma(= \pm 1)$ and an integration path are chosen in such a way to have:

$$
\sigma \Re\left(W\left(\xi_{j}\right)-W\left(\xi_{j+1}\right)\right) \leq 0
$$

for any ordered pair of integration variables (with $\xi_{0}=x$ ). Such a path of integration is then called canonical.

The term $\delta(x)$ appearing in $(2.6)$ and in $(2.7)$ is necessary to ensure all the integrals in (2.5) to converge when $x_{0}$ is a first or a second order pole of $V(x)$ or when solutions (2.4) are to be continued to such poles. Each such a pole $x_{0}$ demands a contribution to $\delta(x)$ of the form $\left(2\left(x-x_{0}\right)\right)^{-2}$ so that $\delta(x)$ collects all of them and its final form depends of course on the corresponding singular structure of $V(x)$.

Note that the effect of introducing the $\delta$-term is completely equivalent to making some change of variable in the SE, a possibility which in this context shall, however, not be discussed in the paper.

In a domain $D_{k}$ of the $x$-plane where the condition (2.8) is satisfied (so called canonical domain) the series in (2.5) defining $\chi_{k}$ is uniformly convergent. $\chi_{k}$ itself satisfies the following initial conditions:

$$
\chi_{k}\left(x_{0}\right)=1 \text { and } \chi_{k}^{\prime}\left(x_{0}\right)=0
$$

corresponding to the equation:

$$
\chi_{k}(x)=1-\frac{\sigma \hbar}{2} \int_{x_{0}}^{x} d y \omega(y) \chi_{k}-\frac{\sigma \hbar}{2} \tilde{q}^{-\frac{1}{2}}(x) \chi_{k}^{\prime}(x)
$$

this function has to obey as a consequence of SE (2.3) and the initial conditions (2.9).

In the canonical domain $D_{k}$ and the sector $S_{k}\left(\subset D_{k}\right)$ where the solution (2.4) is defined the latter has two following basic properties:

$1^{0}$ It can be expanded in $D_{k}$ into a standard semiclassical series obtained by iterating Eq.(2.10) and taking into account the initial conditions (2.9);

$2^{0}$ The emerging semiclassical series is Borel summable in $S_{k}$ to the solution itself.

The solutions (2.4) defined in the above way are known as the fundamental ones [8, 9]. They are pairwise independent and collected into a full set of them they allow to solve any one-dimensional problem. They are distinguished by the property $2^{0}$ above i.e. they are the unique solutions to SE with this property [7]. 


\section{Standard semiclassical expansions}

By a standard semiclassical expansion for $\chi$ we mean the following series:

$$
\begin{aligned}
& \chi(x) \sim C(\hbar) \sum_{n \geq 0}\left(-\frac{\sigma \hbar}{2}\right)^{n} \chi_{n}(x) \\
& \chi_{0}(x)=1 \\
& \chi_{n}(x)=\int_{x_{0}}^{x} d \xi_{n} \tilde{D}\left(\xi_{n}\right) \times \\
& \times \int_{x_{0}}^{\xi_{n}} d \xi_{n-1} \tilde{D}\left(\xi_{n-1}\right) \ldots \int_{x_{0}}^{\xi_{3}} d \xi_{2} \tilde{D}\left(\xi_{2}\right) \int_{x_{0}}^{x} d \xi_{1}\left(\tilde{q}^{-\frac{1}{4}}\left(\xi_{1}\right)\left(\tilde{q}^{-\frac{1}{4}}\left(\xi_{1}\right)\right)^{\prime \prime}+\tilde{q}^{-\frac{1}{2}}\left(\xi_{1}\right) \delta\left(\xi_{1}\right)\right) \\
& n=1,2, \ldots \\
& \tilde{D}(x)=\tilde{q}^{-\frac{1}{4}}(x) \frac{d^{2}}{d x^{2}} \tilde{q}^{-\frac{1}{4}}(x)+\tilde{q}^{-\frac{1}{2}}(x) \delta(x) \\
& C(\hbar)=\sum_{n \geq 0} C_{n}\left(-\frac{\sigma \hbar}{2}\right)^{n}
\end{aligned}
$$

where a choice of a point $x_{0}$ and constants $C_{k}, k=1,2, \ldots$, is arbitrary. However, for the particular $\chi_{k}$ (as defined by (2.5), for example) this choice is of course definite (if $x_{0}$ is given by the lower limit of the integrations in the expansion $(2.5)$ then $C(\hbar) \equiv 1)$. Nevertheless, even in such cases the choice of $x_{0}$ can be arbitrary. Only the constants $C_{k}$ accompanied to the choice are definite depending on the choice [7].

The representation (3.1) is standard in a sense that any other one can be brought to (3.1) by redefinitions of the constants $C_{k}$. Therefore, any semiclassical expansion can be uniquely given by fixing $x_{0}$ and the constants $C_{k}$.

And conversly, multiplying a given semiclassical expansion by an asymptotic series as defined by the last series in (3.1) with other constants $C_{k}, k=1,2, \ldots$, one can obtain any other semiclassical expansion.

We have mentioned above that the semiclassical series for $\chi_{k}$ is Borel summable for $x$ staying in the sector $S_{k}$ where $\chi_{k}$ is defined. In fact it is as such at least inside a circle $\operatorname{Re}\left(\hbar^{-1}\right)^{*}=(2 R)^{-1}$ of the $\hbar$-plane satisfying sufficient conditions of the Watson-SokalNevanlinna (WSN) theorem 10].

Construct now a new semiclassical series by multiplying the one for $\chi_{k}$ by a $\hbar$-dependent constant $C(\hbar)$ with an analytic behaviour at $\hbar=0$. Expand $C(\hbar)$ into a power series in $\hbar$, the latter being simultanuously an asymptotic expansions for the constant. Multiply with this power series the corresponding semiclassical expansion for $\chi_{k}$.

A resulting semiclassical series can be now Borel resummed leading us again to another solution to SE. However, this new solution can have now two representions: the one being the solutions (2.4) multiplied by $C(\hbar)$, and the second being a solution provided by the performed Borel resummation i.e. there is no a priori a necessity for these two representations to coincide.

This is exactly what is observed when a change of variable in SE is performed. 


\section{Change of variable as Borel resummations}

Consider therefore a change of variable in (2.3) putting $y=y(x)$ and assuming $y^{\prime}(x)$ to be meromorphic. Such a change of variable preserves the SE (2.3) if simultanuously we make a substitution: $\Phi(y(x)) \equiv y^{\prime \frac{1}{2}}(x) \Psi(x)$ and $Q(y)$ corresponding to $\Phi(y)$ in its Schrödinger-like equation is given by:

$$
y^{\prime 2}(x) Q(y(x))=q(x)-\hbar^{2}\left(\frac{3}{4} \frac{y^{\prime \prime 2}(x)}{y^{\prime 2}(x)}-\frac{1}{2} \frac{y^{\prime \prime \prime}(x)}{y^{\prime}(x)}\right)
$$

Therefore, the above change of variable provide us with a new potential differing from the old one by the term which depends totaly on $y(x)$. It follows from the form of this term that since $y^{\prime}(x)$ is assumed to be meromorphic this dependence can introduce to the new potential at most second order poles not cancelling the ones of the original potential $V(x)$ if the latter poles do not depend on $\hbar$. It then follows further that the new second order poles can introduce to the corresponding SG additional sectors and SL's not cancelling the old ones built around the old infinite points of the actions. The old sectors of course change their boundaries and enviroments (having possibly as their neighbours some new sectors).

Consider now therefore the old sector $S_{k}$ and its new modified form $\tilde{S}_{k}$. Both the sectors have a common part containing $x_{0}$ at its boundary. Using $\Phi(y)$ and $Q(y)$ we can construct in the $\tilde{S}_{k}$ a solution $\tilde{\Psi}_{k}(x)$ to $\mathrm{SE}(2.3)$. Namely, we have:

$$
\begin{gathered}
\tilde{\Psi}_{k}=\left(y^{\prime 2} \tilde{Q}(y(x))\right)^{-\frac{1}{4}} e^{\frac{\sigma}{\hbar} \int_{x_{i}}^{x} \sqrt{y^{\prime 2}(\xi) \tilde{Q}(y(\xi))} d \xi} \tilde{\chi}_{k}(y(x)) \\
k=1,2, \ldots
\end{gathered}
$$

where $\tilde{\chi}_{k}(y)$ is constructed according to (2.5) - (2.7) by making there substitutions: $x \rightarrow y(=y(x)), \delta(x) \rightarrow \tilde{\delta}(y), \tilde{q}(x) \rightarrow \tilde{Q}(y), \omega(x) \rightarrow \tilde{\omega}(y), W(x) \rightarrow \tilde{W}(y)$ and $x_{0} \rightarrow y_{0}\left(=y\left(x_{0}\right)\right)$.

Note that the new second order poles introduced to $(4.2)$ by $y^{\prime}(x)$ being not present in the original potential $V(x)$ are not real singularities of $\tilde{\Psi}_{k}(x)$. They are only singularities of the representation (4.2).

To the solution (4.2) there correspond a domain $\tilde{D}_{k}$ (an obvious analogue of $D$ given by the inequality (2.8)) in which the solution has the same properties $1^{0}, 2^{0}$ above as the previous ones defined by (2.4)-(2.7). In particular the solutions (4.2) is Borel summable to itself in $\tilde{S}_{k}$

Let us note further that because the sectors $S_{k}$ and $\widetilde{S}_{k}$ have a common part with $x_{0}$ at its boundary then the solutions (2.4) and (4.2) defined in the corresponding sectors have to coincide with each other up to a muliplicative constant $C_{k}$ i.e.

$$
\tilde{\Psi}_{k}(x)=C_{k}(\hbar) \Psi_{k}(x) \quad k=1,2, \ldots
$$

with $C_{k}(\hbar)$ given by

$$
C_{k}(\hbar)=\exp \left[\sigma \hbar \int_{x_{i}}^{x_{0}} \frac{\tilde{\delta}(x)-f(x)}{\sqrt{\tilde{q}(x)}+\sqrt{q(x)+\hbar^{2} \tilde{\delta}(x)-\hbar^{2} f(x)}} d x\right]
$$


where

$$
f(x)=\frac{3}{4} \frac{y^{\prime \prime 2}(x)}{y^{\prime 2}(x)}-\frac{1}{2} \frac{y^{\prime \prime \prime}(x)}{y^{\prime}(x)}
$$

The coefficient $C_{k}$ was calculated by taking a limit $x \rightarrow x_{0}$ on both sides of (4.3).

From (4.3) and (4.4) we get the following relation between $\tilde{\chi}_{k}$ and $\chi_{k}$ :

$\tilde{\chi}_{k}(x)=\left(1+\hbar^{2} \frac{\tilde{\delta}(x)-f(x)}{\tilde{q}(x)}\right)^{\frac{1}{4}} \exp \left[-\sigma \hbar \int_{x_{0}}^{x} \frac{\tilde{\delta}(\xi)-f(\xi)}{\sqrt{\tilde{q}(\xi)}+\sqrt{\tilde{q}(\xi)+\hbar^{2} \tilde{\delta}(\xi)-\hbar^{2} f(\xi)}} d \xi\right] \chi_{k}(x)(4$

Note that the two factors in (4.6) staying in front of $\tilde{\chi}_{k}$ are holomorphic with respect to $\hbar$ at $\hbar=0$.

We shall now show that the solution (4.2) as well as its $\tilde{\chi}_{k}$-function are just the Borel sums of the corresponding semiclassically expanded right hand sights in (4.3) and (4.6), respectively.

This is an immediate consequence of the holomorphicity of the coefficient $C_{k}(\hbar)$ and of the two factors in (4.6) at $\hbar=0$ due to which their semiclassical expansions coincide with their convergent power series expansion in $\hbar$. Therefore, due to our earlier discussion the WSN conditions for Borel summability of the semiclassical series emerging from RHS in (4.3) and (4.6) are satisfied and $\tilde{\Psi}_{k}(x)$ and $\tilde{\chi}_{k}(x)$ are obtained by taking these Borel sums.

\section{Change of variable and exactness of JWKB quantization formulae}

The last result can be done even more appealing by using the following exponential representation for $\tilde{\chi}_{k}(x)$ and $\chi_{k}(x)$ :

$$
\tilde{\chi}_{k}(x)=\exp \left(\int_{x_{0}}^{x} \tilde{\rho}_{k}(\xi) d \xi\right) \quad, \quad \chi_{k}(x)=\exp \left(\int_{x_{0}}^{x} \rho_{k}(\xi) d \xi\right)
$$

so that

$$
\tilde{\rho}_{k}(x)=\frac{\tilde{\chi}_{k}^{\prime}}{\tilde{\chi}_{k}}, \quad \rho_{k}(x)=\frac{\chi_{k}^{\prime}}{\chi_{k}}
$$

and the relation (4.6) takes the form:

$$
\begin{array}{r}
\tilde{\rho}_{k}(x)=\rho_{k}(x)-\sigma \hbar \frac{\tilde{\delta}(x)-f(x)}{\sqrt{\tilde{q}(x)}+\sqrt{q(x)+\hbar^{2} \tilde{\delta}(x)-\hbar^{2} f(x)}}+ \\
+\frac{\hbar^{2}}{4} \frac{\tilde{q}(x)}{q(x)+\hbar^{2} \tilde{\delta}(x)-\hbar^{2} f(x)}\left(\frac{\tilde{\delta}(x)-f(x)}{\tilde{q}(x)}\right)^{\prime}
\end{array}
$$

It follows from (5.2) that both $\tilde{\rho}_{k}(x, \hbar)$ and $\rho_{k}(x, \hbar)$ are Borel summable and from (5.3) that their Borel transforms differ by a function holomorphic on the whole Borel plane if both the functions $f(x)$ and $\tilde{\delta}(x)$ are $\hbar$-independent. In the latter case it is clear that one cannot find such $f(x)$ (the form of $\tilde{\delta}(x)$ has to follow from this of $f(x)$ ) to cause $\tilde{\rho}_{k}(x, \hbar)$ to disappear i.e. one cannot be left in $\tilde{\Psi}_{k}(x)$ with its first two JWKB facors only. This is because 
$\tilde{\rho}_{k}(x, \hbar)$ is singular at $\hbar=0$. However, making $f(x)$ to be also $\hbar$-dependent but choosing it holomorphic at $\hbar=0$ we can achieve a result when the first $n$ terms of the semiclassical expansion of $\tilde{\rho}_{k}(x, \hbar)$ vanish. The latter is possible globally (i.e. independently of $k$ ) since the semiclassical expansions of $\tilde{\rho}_{k}(x, \hbar)$ are $k$-independent (i.e. do not contain any inegration on the $x$-plane, see for example [6]). One of our earlier paper is just a good illustration of this possibility [6] (see also a comment below). However, to achieve the goal of vanishing $\tilde{\rho}_{k}(x, \hbar)$ we have to use $f(x, \hbar)$ being singular at $\hbar=0$ and therefore being expected to satisfy all the necessary conditions of Watson-Sokal-Nevanlinna theorem to be Borel summable. In such a case $f(x, \hbar)$ becomes, similarly to $\tilde{\rho}_{k}(x, \hbar)$, sector dependent i.e. within the class of the Borel summable functions there is no possibilty to define a global $y(x, \hbar)$ which when used as a variable transformation defining $f(x, \hbar)$ provides us with $\tilde{\rho}_{k}(x, \hbar)$ deprived of its $\tilde{\chi}_{k}$-factor for all $k$ simultaneously. In a more obvious way one can conclude this from (5.3) putting there $\tilde{\rho}_{k}(x, \hbar)$ equal to zero and then treating the equation obtained in this way as the differential one for $f(x, \hbar)$ where $\rho_{k}(x, \hbar)$ is given. However, for any two different $k$ 's there are two different $\rho_{k}(x, \hbar)$ 's and in consequence two different solutions for $f(x, \hbar)$ have to emerge.

Summarizing the above discussion we can conclude that the effect of variable changing leading us to the solutions (4.2) can be obtained also as a result of Borel resummations of the standard semiclassical expansions for the solutions (2.5) multiplied by a suitaibly chosen $\hbar$-dependent constants.

A choice of the constants in (4.3) can be even done in such a way to produce simultaneously fundamental solutions for which the series in (2.6) start with an arbitrary high power of $\hbar$ [6]. Such a choice corresponds to a total effect of repeating changes of variable when for each subsequent Schroedinger-like equation a new independent variable is the action i.e. $y^{\prime 2}(x)=\tilde{q}(x, \hbar)$. The 'lacking' powers of $\hbar$ are collected then in $\left(y^{\prime 2}(x, \hbar) \tilde{Q}(x, \hbar)\right)^{\frac{1}{4}}$ and in the corresponding exponential factors of the solutions (2.5). These two factors are then the sources of new JWKB approximations generalizing the conventional ones [6].

Nevertheless, as it follows from the above discussion, there is no such a choice of the constants $C_{k}$ which could cause all the corresponding $\tilde{\chi}_{k}$ 's to be reduced to unity if all the constants as given by (4.4) are to be defined only by one global $f(x, \hbar)$ given on its own by some $y(x, \hbar)$ realizing the underlying change of the $x$-variable.

Some basic conclusion of the latter statement for the possibility to get the exact JWKB formula for energy level quantization is the following.

Consider a quantization of 1-dim quantum systems with the help of the fundamental solutions (it has been described in many of our earlier papers [1, 13, 14, 16]). Let us limit the problem to the case when after a change of the $x$-variable there are only two real turning points $x_{1}, x_{2}$ of $y^{\prime 2}(x, \hbar) \tilde{Q}(x, \hbar)$ whilst the rest of them are complex and conjugated pairwise (we assume $y^{\prime 2}(x, \hbar) \tilde{Q}(x, \hbar)$ and E to be real). We assume also that the problem has been limited to a segment $z_{1} \leq x \leq z_{2}$ at the ends of which $y^{\prime 2}(x, \hbar) \tilde{Q}(x, \hbar)$ has poles. In particular we can push any of $z_{1,2}$ (or both of them) to $\mp \infty$ respectively.

To write the corresponding quantization condition for energy $E$ and to handle simultaneously the cases of second and higher order poles we assume $z_{1}$ to be the second order pole and $z_{2}$ to be the higher ones.

It is also necessary to fix to some extent the closest enviroment of the real axis of the $x$ - plane to draw a piece of SG sufficient to write the quantization condition. To this end we assume $x_{3}$ and $\bar{x}_{3}$ as well as $x_{4}$ and $\bar{x}_{4}$ to be another four turning points and $z_{3}$ and $\bar{z}_{3}$ another two second order poles of $y^{\prime 2}(x, \hbar) \tilde{Q}(x, \hbar)$ closest the real axis. Then a possible piece 
of SG can look as in Fig.1.

There is no a unique way of writing the quantization condition corresponding to the figure. Some possible three forms of this condition can be written as [8]:

$$
\exp \left[\frac{\sigma}{\hbar} \oint_{K}\left(y^{\prime 2} \tilde{Q}(x, \hbar)\right)^{\frac{1}{2}} d x\right]=-\frac{\chi_{1 \rightarrow 3}(\hbar) \chi_{2 \rightarrow \overline{3}}(\hbar)}{\chi_{1 \rightarrow \overline{3}}(\hbar) \chi_{2 \rightarrow 3}(\hbar)}=-\frac{\chi_{1 \rightarrow 4}(\hbar) \chi_{2 \rightarrow \overline{3}}(\hbar)}{\chi_{1 \rightarrow \overline{3}}(\hbar) \chi_{2 \rightarrow 4}(\hbar)}
$$

and $\chi_{k \rightarrow j}(\hbar) k, j=1,2,3,4$ are calculated for $x \rightarrow z_{j}$. The closed integration path $K$ is shown in Fig.1. In the figure the paths $\gamma_{1 \rightarrow 3}, \gamma_{2 \rightarrow 3}$, etc., are the integration paths in the formula (2.5) whilst the wavy lines designate corresponding cuts of the $x$-Riemann surface on which all the FS are defined.

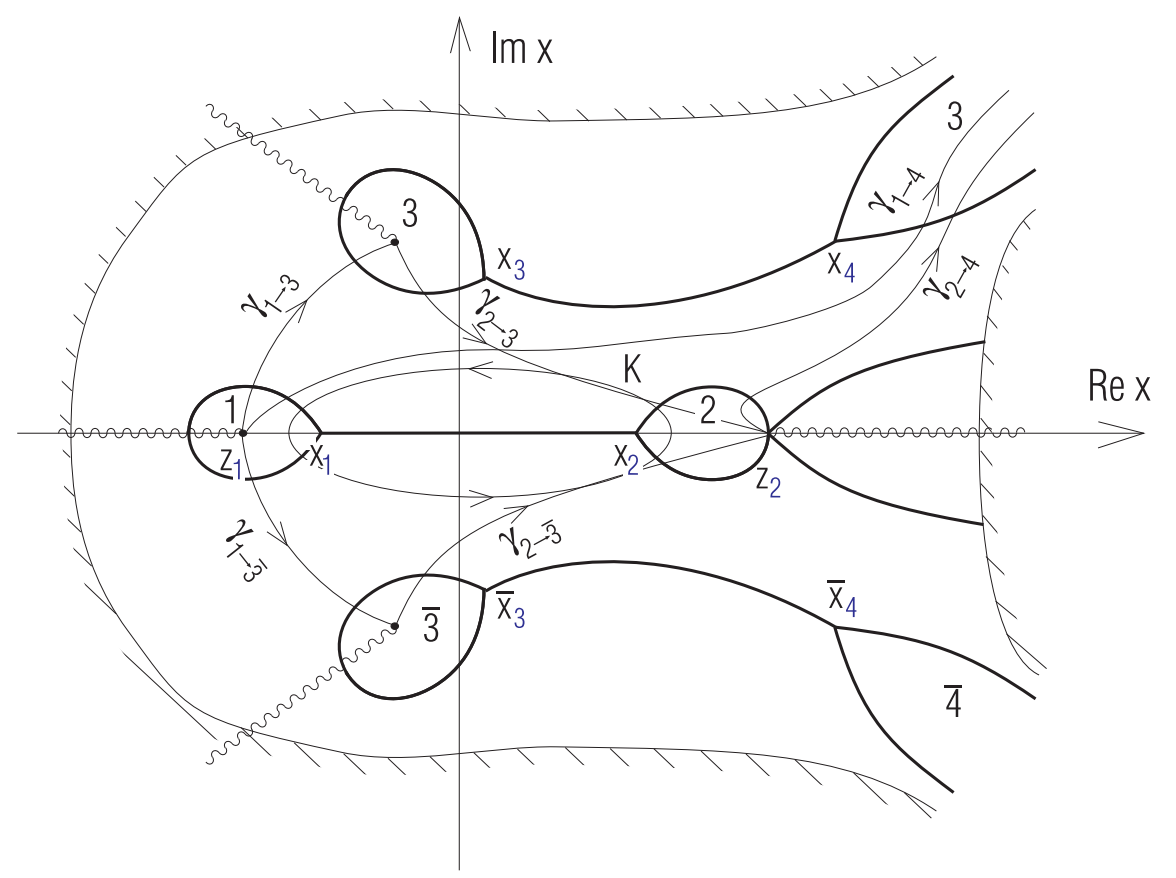

Fig.1 The SG corresponding to general quantization rule (5.4)

The condition (5.4) is exact. Its LHS has just the JWKB form. If we substitude each $\chi_{k \rightarrow j}(\hbar)$ in (5.4) by unity (which these coefficients approach when $\hbar \rightarrow 0$ ) we obtain the wellknown JWKB quantization rule which in general is only an approximation to (5.4).

Now, since there is no an $x$-variable transformation $y(x, \hbar)$ by which all $\chi_{k \rightarrow j}(\hbar)$ in (5.4) could become simultaneously equal to unity the RHS of (5.4) cannot be reduced to unity by any such $y(x, \hbar)$ i.e. the JWKB formula provided in this way by (5.4) is always only an approximation. Some additional symmetry conditions have to be satisfied by the initial $q(x)$ to provide us with such an exact JWKB formula [11].

\section{Conclusions}

To conclude we have shown that the Borel summable fundamental solutions to SE can be modified by appropriate Borel resummations of the latter multiplied by properly chosen $\hbar$ dependent constans. Sometimes effects of such resummations can be recognized as a proper 
change of variable in SE. But the latter can always be considered as an effect of such resummations. This justifies certainly all the improvements and sometimes exact results provided by the change-of-variable procedure applied in JWKB calculations. The latter possibility (i.e. the exact results), however, can realize only due to particular properties of considered potentials reflected in global structures of their respective Stokes graphs [11].

\section{Appendix}

The Maslov method is formulated for an arbitrary linear partial differential equation (LPDE) having as its semiclassical partner a dynamical system with a finite number of degrees of freedom [13]. Maslov's semiclassical theory of solutions to the corresponding LPDE is developed on the 'classical' objects known in the classical mechanics as Lagrangian manifolds [13, 14]. Limited to the one-degree-of-freedom case and to the 1-DSE the Lagrangian manifolds are nothing but the 1-dim classical trajectories in the corresponding 2-dim phase space. Exact solutions to the stationary Schroedinger equation having particular Dirac forms (2.5) can be naturally redefined to live on the Lagrangian manifold (LM) corresponding to a given energy. However, to cover by such a description the whole coordinate domain which the corresponding wave fuctions are defined on, the imaginary time evolution of the classical equations of motion has also to be switched on to take into account so called 'classically forbidden regions'. The emerging LM contains then branches corresponding to the real time motions (performed in classically allowed regions) as well as to the imaginary ones with the imaginary part of the momentum in the latter case playing the role of the classical momentum. Of course, the semiclassical conditions for the considered global wave function are the following: it has to vanish exponentially when $\hbar \rightarrow 0$ in the classically forbidden regions and to oscillate in the classically allowed ones.

Unfortunately, the Dirac representation of these solutions considered as functions of the coordinate cannot be defined globally on the above LM being singular at points where the manifold branches making impossible a matching procedure of the solutions defined on different branches. These singular points are called in general the caustic ones but in the 1-dim case they are known as turning points. Maslov and Fedoriuk's remedy to solve this arising 'connection problem' is to change the coordinate variable around such points into the corresponding momentum i.e. to change the coordinate representation of the wave function into the momentum one preserving the Dirac form of the solution. Assuming the wave function to be normalized, its latter representation can be given formally by the Fourier transformation of the former. In the new representation the wave function is then regular at the coordinate turning points of LM (being on the other hand singular at the emerging momentum turning points). The invers Fourier transformation considered close to a coordinate turning point provides us again with the solution in the coordinate representation given on both the sides of the chosen coordinate turning point. As we have mentioned above the semiclassical limit condition for the latter solution is of course to vanish exponentially (when $\hbar \rightarrow 0$ ) on one side of the turning point and to oscillate on the other. This condition determines the way the local solutions determined on both the sides of each turning point and having the Dirac form are to be matched.

The above idea of matching the solutions on different branches the Lagrangian manifold does not seem to be effective for the exact solutions to SE but it becomes as such when the solutions in their Dirac forms are substituted by their corresponding semiclassical series. 
This is in fact the subject of the original approach of Maslov and collaborators. Namely, in such a case the classically forbidden parts of the solutions disappeared completely (being exponentially small) and the remaining ones are then given uniquely on the classically allowed branches of LM. The matching procedure connects then only two oscillating solutions separated by the corresponding turning point. The underlying Fourier transformation becomes then effectively a point transformation determining the connection. As it is well known [13] such an semiclassical wave function continued through a turning point on LM changes its phase by \pm 1 . (These changes are controlled in general by so called Maslov indeces). Synthetically the whole operation is performed with the help of the Maslov canonical operator [13].

It is easy to note however that the necessity to use Fourier transformation diappears if there are possibilities to avoid somehow turning (caustic) points on the way the wave function is continued on. This can be achieved for example by enlarging the number of dimensions the problem is formulated in. The complexification of the problem is the one of such ways to be used [15]. In the 1-dim case this can be done effectively and without appealing directly to the semiclassical series expansions by defining the problem on the complex coordinate plane and utilizing the notions of Stokes graphs and fundamental solutions. In comparison with Maslov's approach the complex coordinate plane (in fact the latter is rather a Riemann surface) corresponds to the complex Lagrangian manifold endowed with the coordinate charts collected of all canonical domains defined by the corresponding Stokes graph. To each canonical domain a fundamental solution is attached having the corresponding domain as the maximal one where its semiclassical expansion as given by (3.1) is valid. There is no necessity to construct and to use the Maslov canonical operator to continue (analytically) the fundamental solutions and to match them in any domain of the plane. The Maslov indeces gained by the fundamental solutions on the way of their analytical continuations are provided by crossed cuts of the corresponding Riemann surface. Therefore using the fundamental solution method in the 1-dim problems is completely equivalent to the corresponding Maslov one in the semiclassical regime of the problem but it has many obvious advantages over the latter with their use as the exact solutions to SE being the first one. Other important properties of the method have been mentioned and used in the main body of this as well as other papers [6, 0, 8, 9, 11].

\section{References}

[1] Langer R.E. , Phys. Rev. 51 (1937) 669-76

[2] Berry M.V. and Mount K.E. , Rep. Prog. Phys. 35 (1972) 315-397

[3] Fröman N. and Fröman P.O. , JWKB Approximation. Contribution to the Theory, NorthHolland, Amsterdam 1965

[4] Rozenzweig C. and Krieger J.B., J. Math. Phys. 9 (1968) 849-860

[5] Krieger J.B., J. Math. Phys. 10 (1969) 1455-1458

[6] Giller S., J. Phys. A: Math. Gen. 21 (1988) 909 
[7] Giller S. and Milczarski P., "Borel summable solutions to 1-dim Schrödinger equation" , quant-ph/9801031

[8] Giller S., Acta Phys. Pol. B23 (1992) 457-511

[9] Giller S., Acta Phys. Pol. B21 (1990) 675-709

[10] Watson G.N., Philos. Trans. Soc. London Ser.A 211 (1912) 279

Sokal A.D., J. Math. Phys. 21 (1980) 261-263

Nevanlinna F., Ann. Acad. Sci. Fenn. Ser. A 12, No.3 (1918-19)

[11] Milczarski P. and Giller S., "Some Results on Conventional and Supersymmetric JWKB Formulae and Their Relation to Shape Invariance Property of Potentials", to be published

[12] Bose A K, Nuo. Cim. 32 (1964) 679

[13] Maslov V.P. and Fedoriuk M.V., Semi-classical Approximation in Quantum Mechanics, Reidel Pub. Com.: Dordrecht, Boston, London 1981

[14] Arnold V.I., Mathematical Methods of Classical Dynamics, Springer: New York, 1978.

[15] Brihaye Y., Giller S., Gonera C. and Kosinski P., Complex Maslov Method Applied to Integrable Hamiltonian Systems in: "GROUP21, Physical Applications and Mathematical Aspects of Geometry, Groups, and Algebras", Volume 1, Editors: H.D. Doebner, W. Scherer, P. Nattermann, World Scientific, Singapore, 1997, p. 411 proceedings of XXI International Colloquium on Group Theoretical Methods in Physics (Group21), 15 - 20 July 1996, Goslar, Germany

[16] Giler S., J. Phys. A: Math. Gen. 22 (1989) 2965-2990 